\title{
miR-409-3p is reduced in plasma and islet immune infiltrates of NOD diabetic mice and is differentially expressed in people with type 1 diabetes
}

\author{
Giuliana Ventriglia $^{1,2,3}$. Francesca Mancarella ${ }^{1,2} \cdot$ Guido Sebastiani $^{1,2} \cdot$ Dana P. Cook $^{3}$ - Roberto Mallone ${ }^{4}$ \\ Chantal Mathieu ${ }^{3} \cdot$ Conny Gysemans ${ }^{3}$. Francesco Dotta ${ }^{1,2}$
}

Received: 9 April 2019 / Accepted: 30 August 2019/Published online: 28 October 2019

(C) Springer-Verlag GmbH Germany, part of Springer Nature 2019

\begin{abstract}
Aims/hypothesis MicroRNAs (miRNAs) are a novel class of potential biomarkers emerging in many diseases, including type 1 diabetes. Here, we aim to analyse a panel of circulating miRNAs in non-obese diabetic (NOD) mice and individuals with type 1 diabetes.

Methods We adopted standardised methodologies for extracting miRNAs from small sample volumes to evaluate a profiling panel of mature miRNAs in paired plasma and laser-captured microdissected immune-infiltrated islets of recently diabetic and normoglycaemic NOD mice. Moreover, we validated the findings during disease progression and remission after anti-CD3 therapy in NOD mice, as well as in individuals with type 1 diabetes.

Results Plasma levels of five miRNAs were downregulated in diabetic vs normoglycaemic mice. Of those, miR-409-3p was also downregulated in situ in the immune islet infiltrates of diabetic mice, suggesting an association with disease pathogenesis. Targetprediction tools linked miR-409-3p to immune- and metabolism-related signalling molecules. In situ miR-409-3p expression correlated with insulitis severity, and $\mathrm{CD}^{+}$central memory T cells were found to be enriched in miR-409-3p. Plasma miR-409$3 p$ levels gradually decreased during diabetes development and improved with disease remission after anti-CD3 antibody therapy. Finally, plasma miR-409-3p levels were lower in people recently diagnosed with type 1 diabetes compared with a non-diabetic control group, and levels were inversely correlated with $\mathrm{HbA}_{1 \mathrm{c}}$ levels.

Conclusions/interpretation We propose that miR-409-3p may represent a new circulating biomarker of islet inflammation and type 1 diabetes severity.
\end{abstract}

Keywords Anti-CD3 therapy $\cdot$ Biomarker $\cdot$ Inflammation $\cdot$ MicroRNA $\cdot$ Progression $\cdot$ Type 1 diabetes

Giuliana Ventriglia and Francesca Mancarella are joint first authors. Conny Gysemans and Francesco Dotta are joint senior authors.

Electronic supplementary material The online version of this article (https://doi.org/10.1007/s00125-019-05026-1) contains peer-reviewed but unedited supplementary material, which is available to authorised users.

Francesco Dotta

francesco.dotta@unisi.it

Diabetes Unit, Department of Medicine, Surgery and Neurosciences, University of Siena, V.le Bracci, 16 - 53100 Siena, Italy

2 Fondazione Umberto Di Mario ONLUS c/o Toscana Life Sciences, Siena, Italy

3 Clinical and Experimental Endocrinology (CEE), Katholieke Universiteit Leuven (KU LEUVEN), Leuven, Belgium

4 Inserm, U1016, CNRS, UMR8104, Paris Descartes University, Sorbonne Paris Cité, Cochin Institute, Paris, France

\section{Abbreviations}

aCD3 Anti-CD3

LCM Laser-captured microdissected

mAb Monoclonal antibody

miRNA MicroRNA

NOR Non-obese resistant

SOP Standard operating procedure

TCM Central memory T cell

TEM Effector memory T cell

3'-UTR 3'-Untranslated region

\section{Introduction}

Circulating biomarkers for screening, diagnosis and monitoring type 1 diabetes include blood glucose level, $\mathrm{HbA}_{1 \mathrm{c}}$ and 


\section{Research in context}

\section{What is already known about this subject?}

- Circulating microRNAs (miRNAs) have been shown to be altered in the plasma of individuals with type 1 diabetes

- Circulating miRNA profiles are associated with hyperglycaemia in people with type 1 diabetes

- Circulating miRNAs are deregulated in plasma-derived exosomes from people with type 1 diabetes

\section{What is the key question?}

- Can we identify circulating plasma-borne miRNAs that mirror in situ pancreatic inflammation in type 1 diabetes?

\section{What are the new findings?}

- $\quad$ miR-409-3p was reduced in plasma of recently diabetic NOD mice

- $\quad$ miR-409-3p was reduced in pancreatic immune infiltrates of recently diabetic NOD mice and correlated with insulitis severity

- $\quad$ miR-409-3p was differentially expressed in the plasma of individuals with recently diagnosed type 1 diabetes

\section{How might this impact on clinical practice in the foreseeable future?}

- Circulating plasma-borne microRNAs may be clinically applicable biomarkers that could help to stage disease progression and to stratify individuals with type 1 diabetes

autoantibody measurements. They are often detected only during the late stages of disease development, when most beta cells are destroyed. Although genotyping and islet autoantibodies perform relatively well at predicting future type 1 diabetes, it remains challenging to accurately monitor islet inflammation during disease progression.

MicroRNAs (miRNAs), a class of small non-coding RNAs about 19-24 nucleotides long and processed from hairpin precursors by the drosha ribonuclease III (DROSHA)-DGCR8 microprocessor complex subunit (DGCR8) complex and dicer 1, ribonuclease III (DICER) [1], have been proposed as circulating biomarkers. miRNAs modulate gene expression by binding to complementary sites within 3'-untranslated regions (3'-UTRs) of target mRNAs, leading to translational repression or to mRNA degradation $[2,3]$. Mature miRNAs can be either functional, to guide translational repression of target mRNAs, or released in extracellular fluids $[4,5][6]$. The high stability of miRNAs in tissues and extracellular fluids and the possibility that alterations in cell-free circulating miRNAs may be paralleled by a dysregulation in a cellular subset support their potential use as biomarkers. The biological role of circulating miRNAs as mediators of cell-to-cell crosstalk is yet to be defined. Nonetheless, alterations in miRNA expression in body fluids can be detected before conventional biomarkers and have been associated with different pathophysiological conditions, such as cancer [7], autoimmune diseases $[8,9]$, and cardiovascular [10] and metabolic disorders, even though detailed information on their specific cells of origin is lacking in most reports.
Recent studies analysed miRNA expression profiles in serum or plasma from individuals with type 1 diabetes in order to explore new approaches to monitor the development, progression or resolution of autoimmune diabetes [11-16]. Altered miRNA signatures were detected in these studies, and levels of specific circulating miRNAs correlated with beta cell function or other clinical variables in some of these studies. However, owing to the obvious difficulties related to tissue procurement and collection, none of the published studies identified altered miRNAs in both the circulation and the diseased organ, not even in the non-obese diabetic (NOD) mouse model of autoimmune diabetes.

We adopted standardised methodologies for extracting miRNAs from small sample volumes with the aim of evaluating a profiling panel of mature miRNAs in paired plasma and in laser-captured microdissected (LCM) immuneinfiltrated islets of recently diabetic and normoglycaemic NOD mice.

\section{Methods}

Experimental workflow We analysed an miRNA profiling panel in plasma of age-matched (same week of age) normoglycaemic and recently diabetic NOD mice $(n=5$ each) and validated the findings in matched LCM islet endocrine cells and immune infiltrates (key resources are shown in electronic supplementary materials [ESM] Table 1). Differentially expressed miRNAs were further investigated: (1) for in situ 
expression according to insulitis severity; (2) in the cellular subsets particularly enriched for the miRNA(s) of interest; (3) in plasma samples from an additional group of recently diabetic $(n=12)$, age-matched normoglycaemic $(n=25)$, 4week-old $(n=14)$ and 8 -week-old $(n=14)$ prediabetic NOD mice; and (4) for changes in expression after an in situ targeted therapeutic intervention. Finally, we analysed plasma miR409-3p expression in two independent human cohorts: a first cohort ('Cohort 1') of non-diabetic individuals (control group) ( $n=25)$, autoantibody-negative relatives of individuals with type 1 diabetes $(n=9)$, and individuals recently diagnosed with type 1 diabetes $(n=18)$, whose blood samples were collected and processed following a strict standard operating procedure (SOP); and a second, historical, cohort group ('Cohort 2') of individuals without diabetes (control group) ( $n=17)$, individuals recently diagnosed with type 1 diabetes ( $n=23)$ and individuals with longstanding type 1 diabetes ( $n$ $=13)$, as well as individuals with rheumatoid arthritis $(n=18)$.

Animals NOD mice were housed and inbred in the KU Leuven animal facility (Leuven, Belgium) since 1989 and kept under semi-barrier conditions, as described [17, 18]. Female mice were screened for diabetes by glycosuria (Diastix Reagent Strips; Bayer, Leverkusen, Germany) and venous blood glucose levels (Accu-Chek; Roche Diagnostics, Vilvoorde, Belgium). Mice were diagnosed as diabetic when positive for glycosuria and for two consecutive blood glucose measurements $>11.1 \mathrm{mmol} / \mathrm{l}$. Recently diabetic NOD mice were 12 to 22 weeks old; age-matched normoglycaemic animals were used as controls. Non-obese resistant (NOR) mice were obtained from the Jackson Laboratory (Bar Harbor, ME, USA) and killed at around 21 weeks of age. NOD mice of 4 and 8 weeks of age were used to evaluate miR-409-3p expression during the prediabetic phase.

For anti-CD3 (aCD3) monoclonal antibody (mAb) therapy, recently diabetic NOD mice were treated for 7 consecutive days with increasing i.v. doses (day $1,0.42 \mu \mathrm{g}$; day 2, 1.67 $\mu \mathrm{g}$; days 3-7, $4.46 \mu \mathrm{g}$ per mouse) of anti-mouse CD3 mAb (clone 145-2C11; BioXCell, West Lebanon, NH, USA) (key resources are shown in ESM Table 2). Blood was collected by heart puncture or submandibular bleeding and centrifuged at $2000 \mathrm{~g}$ for $10 \mathrm{~min}$ at $4^{\circ} \mathrm{C}$ to obtain plasma. Collected plasma was immediately stored at $-80^{\circ} \mathrm{C}$. Mice were bred and housed according to protocols approved by the Katholieke Universiteit Leuven Animal Care and Use Committee (Leuven, Belgium; project number 116/2015) and experiments complied with EU Directive 2010/63/EU for animal experiments.

Human donors In Cohort 1, individuals with recently diagnosed type 1 diabetes ( $<1$ year of diagnosis; $n=18$ ) and autoantibody-negative non-diabetic first-degree relatives of individuals with type 1 diabetes $(n=9)$ and without diabetes
( $n=25)$ were recruited in the outpatient diabetes centre, University Hospital UZ Leuven (Leuven, Belgium), and at the diabetes outpatient unit, Siena University Hospital (Siena, Italy) (Table 1). Informed written consent was obtained from all individuals enrolled in the study, which was approved by the local ethics committee (Comitato Etico Regionale per la Sperimentazione Clinica della Toscana Area Vasta Sud Est - cod. INNODIA01).

All non-diabetic individuals were negative for islet autoantibodies and for signs of ongoing endocrine dysfunction or of other autoimmune diseases. A SOP was followed to collect plasma samples (see 'Human donors - blood collection procedure' section in ESM Methods). Additional plasma samples were analysed; these were derived from Cohort 2 composed of non-diabetic individuals (control group) $(n=17)$, individuals recently diagnosed with type 1 diabetes $(<1$ year of diagnosis, $n=23)$ and individuals with longstanding type 1 diabetes ( $>2$ years of diagnosis, $n=13$ ), in addition to individuals with rheumatoid arthritis (ESM Table 3). All individuals with type 1 diabetes were positive for at least one autoantibody. Autoantibodies were analysed as described previously [19].

Plasma RNA extraction Total RNA, including miRNAs, was extracted from $50 \mu \mathrm{l}$ NOD mouse plasma or from $100 \mu \mathrm{l}$ human plasma using miRNeasy kit (Qiagen, Hilden, Germany) (see ESM Methods).

Extracellular circulating miRNA profiling and data analysis miRNA profiling was performed using TaqMan miRNA array rodent microfluidic cards (Panel A v2.1; Life Technologies, Carlsbad, CA, USA). Megaplex reverse transcriptase reaction and miRNA arrays were performed according to the manufacturer's protocols (Life Technologies). Samples were checked for housekeeping stability and haemolysis rate (see ESM Methods; ESM Fig. 1a,b).

LCM and insulitis grading Islet endocrine cells and juxtaposed infiltrating immune cells were microdissected separately as described previously [17]. LCM islet endocrine cells and immune infiltrates were pooled separately according to insulitis score: score 0 (no infiltration) and 1 (peri-insulitis) were pooled together; tissues with score 2 (infiltration in $<50 \%$ of the islet area) and 3 (infiltration in $\geq 50 \%$ of the islet area) were pooled separately (see ESM Methods; ESM Fig. 2).

Cell staining, flow cytometry and cell sorting Single cell suspensions were prepared from pancreases in digestion medium (RPMI medium + 5\% FCS, $2 \mathrm{mmol} / 1 \mathrm{~L}$-glutamine, 0.05 $\mathrm{mmol} / 1 \beta$-mercaptoethanol, $100 \mathrm{U} / \mathrm{ml}$ penicillin, $100 \mathrm{mg} / \mathrm{ml}$ streptomycin, $1 \mathrm{mg} / \mathrm{ml}$ collagenase-VIII and $0.02 \mathrm{mg} / \mathrm{ml}$ DNase-I; Life Technologies) for $15 \mathrm{~min}$ in a shaking incubator at $150 \mathrm{rpm}$ at $37^{\circ} \mathrm{C}$. The phenotype analysis was performed with flow cytometry by staining the cells with anti-CD4, 
Table 1 Main characteristics of the non-diabetic control group, first-degree relatives and individuals with type 1 diabetes in Cohort 1

\begin{tabular}{|c|c|c|c|c|}
\hline \multirow[t]{2}{*}{ Characteristic } & \multicolumn{3}{|c|}{ Control participants } & \multirow[t]{2}{*}{ Type 1 diabetes } \\
\hline & Unrelated $^{\mathrm{a}}$ & UFM & Total & \\
\hline$n$ & 25 & 9 & 34 & 18 \\
\hline Clinical site (Siena: Leuven), $n: n$ & $13: 12$ & $8: 1$ & $21: 13$ & $3: 15$ \\
\hline Age, years & $28.0 \pm 7.0$ & $31.0 \pm 8.8$ & $29.0 \pm 7.3$ & $31.0 \pm 10.2$ \\
\hline Male sex (female sex), $n$ & $12(13)$ & $1(8)$ & $13(21)$ & $14(4)$ \\
\hline Fasting blood glucose, $\mathrm{mmol} / \mathrm{l}$ & ND & $\mathrm{ND}$ & ND & $11.9 \pm 6.8$ \\
\hline Insulin dose, U/day & NA & NA & NA & $45 \pm 12.0$ \\
\hline BMI, $\mathrm{kg} / \mathrm{m}^{2}$ & ND & $\mathrm{ND}$ & ND & $21 \pm 2.0$ \\
\hline $\mathrm{HbA}_{1 \mathrm{c}}, \%$ & ND & $\mathrm{ND}$ & ND & $13.3 \pm 2.1$ \\
\hline $\mathrm{HbA}_{1 \mathrm{c}}, \mathrm{mmol} / \mathrm{mol}$ & ND & ND & ND & $120.9 \pm 23.3$ \\
\hline C-peptide, nmol/1 & ND & ND & ND & $0.07 \pm 0.05$ \\
\hline $\mathrm{ICA}^{+}, n$ & 0 & 0 & 0 & 6 \\
\hline $\mathrm{IAA}^{+}, n$ & 0 & 0 & 0 & 2 \\
\hline $\mathrm{GADA}^{+}, n$ & 0 & 0 & 0 & 14 \\
\hline IA- $2 \mathrm{~A}^{+}, n$ & 0 & 0 & 0 & 7 \\
\hline $\mathrm{ZnT} \mathrm{A}^{+}, n$ & 0 & 0 & 0 & 4 \\
\hline
\end{tabular}

Data are shown as mean \pm SD unless stated otherwise

${ }^{\text {a }}$ Control participant without familiality for type 1 diabetes

GADA, GAD autoantibody; IAA, insulin autoantibody; IA-2A, insulinoma-associated 2A autoantibody; ICA, islet cell autoantibody; NA, not applicable; ND, not determined; UFM, unaffected family member; ZnT8A, zinc transporter 8 autoantibody
-CD8, -CD44 and -CD62L mAbs, all from eBioscience (San Diego, CA, USA) (ESM Fig. 3). Cells were sorted directly into Trizol-LS (Life Technologies). RNA was extracted, quantified and quality controlled as above.

miRNA and mRNA single-assay real-time PCR To validate miRNA expression in the reverse transcription (RT)-PCR single-assay reactions, $1 \mathrm{ng}$ of RNA extracted from LCM islet immune infiltrates or flow-sorted immune cells, RNA extracted from $50 \mu \mathrm{l}$ NOD mouse plasma or $100 \mu$ l human plasma was reverse transcribed using the Megaplex protocol with preamplification, and analysed using real-time PCR through specific TaqMan miRNA assays in 96 well plates (all from Life Technologies). To analyse mRNA expression from LCM islet endocrine cells and immune infiltrates, reverse transcription reactions were performed using ImProm-II RT (Promega, Madison, WI, USA) and TaqMan gene expression assays (Life Technologies), adopting a pre-amplification step following the manufacturer's protocol (see ESM Methods).

miR-409-3p absolute quantification Tenfold serial dilutions from $10^{-2}$ to $10^{-8} \mathrm{nmol} / 1$ of the synthetic miR-409-3p (mirVana miRNA mimic, Life Technologies) were reverse transcribed and assayed in parallel with RNA extracted from human plasma samples (ESM Fig. 4). Single-assay real-time PCR reactions were carried out as reported above (see ESM Methods).
Prediction and pathway enrichment analysis of miRNA targets Putative targets of miR-409-3p, as well as pathway enrichment analysis, were investigated employing miRWalk v.3.0 (http://mirwalk.umm.uni-heidelberg.de). The results were visualised in Cytoscape v.3.6.1. using the ClueGo plugin $[20,21]$.

Statistical Analysis Statistical analyses were performed using GraphPad Prism Version 7.00 (GraphPad Software, La Jolla, CA, USA). Two-tailed Mann-Whitney $U$ tests were used for comparisons between groups; two-tailed Wilcoxon signedrank tests were used for comparisons between paired samples. Data are reported as mean \pm SEM. For all analyses, a $p$ value of $\leq 0.05$ was considered significant. Randomisation and blinding were not carried out.

\section{Results}

A distinct signature of five unique downregulated miRNAs is identified in plasma of recently diabetic mice Expression profiling of 384 miRNAs was performed on five plasma samples obtained from age-matched normoglycaemic and recently diabetic NOD mice (between 12 and 22 weeks of age). The expression of the 182 mature miRNAs that were detected in at least one sample for each group is depicted in the heatmap hierarchical clustering tree (Fig. 1a). We identified seven 

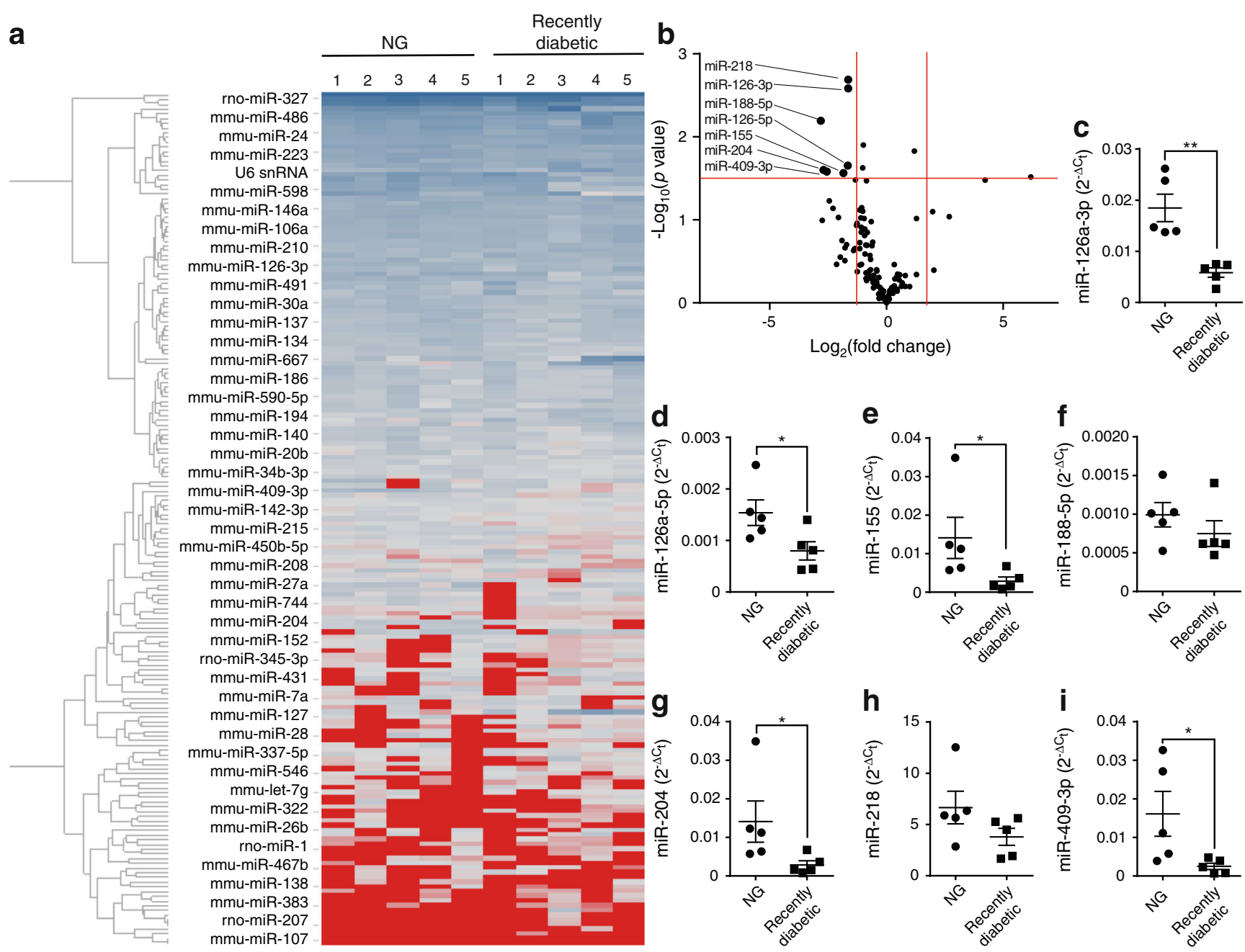

Fig. 1 Circulating miRNA profiling of plasma samples deriving from recently diabetic and normoglycaemic NOD mice. (a) Hierarchical clustering heatmap showing colour-coded expression levels of miRNAs (blue, high expression; red, low expression) in plasma from recently diabetic and age-matched normoglycaemic NOD mice. (b) Volcano plot showing changes in miRNA levels between recently diabetic and normoglycaemic NOD mice. Levels in normoglycaemic mice were set at 0 . Red lines indicate fold change and $p$ value cut-offs. The larger data

points indicate differentially expressed miRNAs. (c-i) Single-assay realtime RT-PCR validation of differentially expressed miRNAs was performed in the same plasma samples as those used for the miRNA screening. Values are expressed as $2^{-\Delta \mathrm{C}_{\mathrm{t}}}$. Data are presented as mean $\pm \mathrm{SEM} ; *$ $\leq 0.05, * * p \leq 0.01$, Mann-Whitney $U$ test. mmu, Mus musculus; NG, normoglycaemic; rno, Rattus norvegicus

differentially expressed miRNAs (miR-126a-3p, miR-126a5p, miR-155, miR-188-3p, miR-204, miR-218 and miR-409$3 p)$. All of them were downregulated in the plasma of diabetic vs normoglycaemic mice (Fig. $1 \mathrm{~b}$ and ESM Fig. 5). Using single-assay real-time PCR, five out of seven miRNAs were validated as differentially expressed (miR-126a-3p, miR-126a5p, miR-155, miR-204 and miR-409-3p) (Fig. 1c-i).

miR-409-3p is reduced in pancreatic immune infiltrates of NOD mice In order to investigate whether these miRNA alterations were also present in matched pancreas samples, LCM was performed to separately capture islet endocrine cells and juxtaposed immune infiltrates from the same mice. miR-126a$3 p$, miR-126a-5p, miR-155 and miR-204 were not differentially expressed in islet endocrine cells captured from diabetic vs normoglycaemic mice nor in LCM juxtaposed immune infiltrates (ESM Fig. 6a-d). Interestingly, miR-409-3p was significantly downregulated in LCM immune infiltrates captured from recently diabetic vs normoglycaemic NOD mice, while no significant differential expression was detected in islet endocrine cells (Fig. 2a,b).

To investigate the potential target genes of miR-409-3p, we employed a conservative strategy by adopting the online target-prediction tool miRWalk. This resulted in three modules that were enriched with the following functions: immunerelated pathways; metabolic pathways; and vesicle transport and cell adhesion. The metabolic module was the most significant, with 19 pathways involved (ESM Table 4). Major immune genes were identified in the immune module, such as Pten, Mapk1, Nfatc1, Nfatc2, Ccr5 and Il6ra. Additionally, 
a

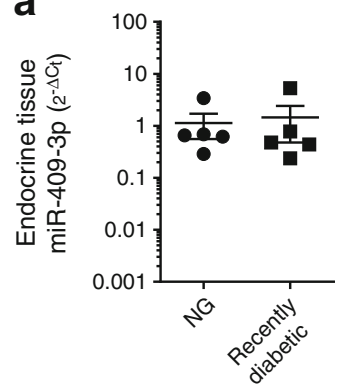

Fig. 2 In situ miR-409-3p expression analysis. Expression analysis of miR-409-3p in (a) LCM-captured islet endocrine cells from normoglycaemic $(n=5)$ and recently diabetic NOD mice $(n=5)$ (all islets had an insulitis score of 2-3) and (b) LCM-captured islet-juxtaposed immune infiltrates from normoglycaemic $(n=5)$ and recently diabetic NOD mice $(n=5)$. Values are expressed as $2^{-\Delta \mathrm{C}_{t}}$ plotted on a $\log 10$ scale. Data are presented as mean \pm SEM. $* * p \leq 0.01$ MannWhitney U test NG, normoglycaemic

target genes (e.g. Gad2) and pathways (e.g. antigen processing and presentation genes, $T$ cell receptor signalling pathway and chemokine signalling pathway) known to play a role in type 1 diabetes were detected (Fig. 3).

miR-409-3p expression in islet immune infiltrates correlates with insulitis severity Based on the resulting miRNA-target interaction network linking miR-409-3p mainly to immune signalling molecules, we hypothesised a potential association of miR409-3p expression with insulitis severity. Therefore, we microdissected islet immune infiltrates from five new pairs of recently diabetic and age-matched normoglycaemic NOD mice and separated them based on their insulitis score (either 1 for mild insulitis or $2 / 3$ for severe insulitis) (Fig. 4a,b). The decreased level of miR-409-3p in plasma (Fig. 4c) and immune infiltrates (Fig. 4d) from this additional group of recently diabetic mice compared with normoglycaemic animals confirmed the results of the previous analysis. By separating the islet immune infiltrates in the normoglycaemic mice by insulitis score, we observed a significant downregulation of miR-409-3p expression in severe insulitis vs mild insulitis (Fig. 4e). Moreover, miR-409$3 p$ expression in immune infiltrates with score $2 / 3$ in the normoglycaemic mice was comparable to those with score $2 / 3$ in recently diabetic mice, further confirming that in situ miR-409$3 p$ expression was linked to insulitis severity (Fig. 4e).

miR-409-3p expression in plasma gradually decreases during autoimmune diabetes progression and is mainly enriched in $\mathrm{CD}^{+}$central memory T cells We further validated the expression of miR-409-3p in plasma samples derived from a new group of recently diabetic NOD mice, age-matched normoglycaemic NOD mice, age-matched diabetes-resistant NOR mice and 4 and 8 week old NOD mice, with various degrees of islet infiltration (Fig. 5a). The analyses confirmed the lower expression of miR-409-3p in plasma from diabetic

vs normoglycaemic mice (Fig. 5b). Furthermore, miR-409-3p expression was already downregulated in plasma of 8-weekold vs 4-week-old mice and progressively decreased until disease onset. Of note, age-matched normoglycaemic NOR mice retained: (1) similar miR-409-3p levels to those of 8-week-old NOD mice; and (2) higher miR-409-3p levels vs age-matched normoglycaemic NOD mice (Fig. 5b). Thus, higher miR-409$3 p$ levels were inversely associated with insulitis severity in NOD mice (Fig. 5a). Additionally, miR-409-3p expression levels inversely correlated with blood glucose levels, indicating a link with disease progression (ESM Fig. 7).

To identify the cellular source of in situ miR-409-3p expression, we purified the most abundant immune-cell subsets from dissected samples of pancreas from longstanding normoglycaemic NOD mice by flow cytometry according to established surface markers [22]. The major cell type in human autoimmune infiltrates is the lymphocyte, with a dominance of $\mathrm{CD} 8^{+}$over $\mathrm{CD} 4^{+} \mathrm{T}$ cells [23-25]. Here, we also studied the $\mathrm{CD} 4^{+}$and $\mathrm{CD} 8^{+} \mathrm{T}$ cell frequencies both in peripheral blood and in pancreas during disease progression in the NOD mouse model. First, we confirmed the predominance of $\mathrm{CD}^{+}$over $\mathrm{CD} 4^{+} \mathrm{T}$ cells in the pancreas (data not shown). We did not observe relevant changes in $\mathrm{CD} 4^{+} \mathrm{T}$ cell frequencies during disease progression (Fig. $5 \mathrm{c}$,d). Moreover, we noticed that the proportion of $\mathrm{CD}^{+} \mathrm{T}$ cells, particularly the $\mathrm{CD} 8^{+}$effector memory $\mathrm{T}$ (TEM; CD $44^{+} \mathrm{CD} 62 \mathrm{~L}^{-}$) cells, gradually increased in the periphery and especially in the pancreas, with beta cell mass waning (Fig. 5e,f). More interestingly, the proportion of $\mathrm{CD}^{+}$central memory $\mathrm{T}$ (TCM; $\mathrm{CD} 44^{+} \mathrm{CD} 62^{+}$) cells decreased in both periphery and pancreas during disease progression (Fig. 5e,f), paralleling circulating miR-409-3p decrease.

Based on a previously reported association of miR-409-3p with human memory $\mathrm{T}$ cells [26], we focused on both isletassociated $\mathrm{CD} 4^{+}$and $\mathrm{CD} 8^{+}$TEM and TCM cells and assessed miR-409-3p enrichment in these immune subsets. The expression of miR-409-3p was significantly enriched in isletassociated $\mathrm{CD}^{+}$over $\mathrm{CD} 4^{+} \mathrm{T}$ cells, more specifically in TCM vs TEM subsets (Fig. 5g).

Plasma miR-409-3p expression improves after disease intervention therapy with anti-CD3 antibody We treated recently diabetic NOD mice with increasing i.v. doses of aCD3 mAbs, which have been shown to change the anti-islet immune response and stabilise disease progression [27-29]. We assessed plasma miR-409-3p at disease onset (day 0 ) and 7 days after the last aCD3 injection (day 14) (Fig. 6a). At day 14, plasma miR-409-3p levels were increased as compared with day 0 in aCD3-treated mice, while no significant changes were observed in untreated mice (Fig. 6b,c). Additionally, miR-409$3 p$ levels did not correlate with blood glucose levels upon $\mathrm{aCD} 3$ treatment, thus suggesting an intrinsic association with 


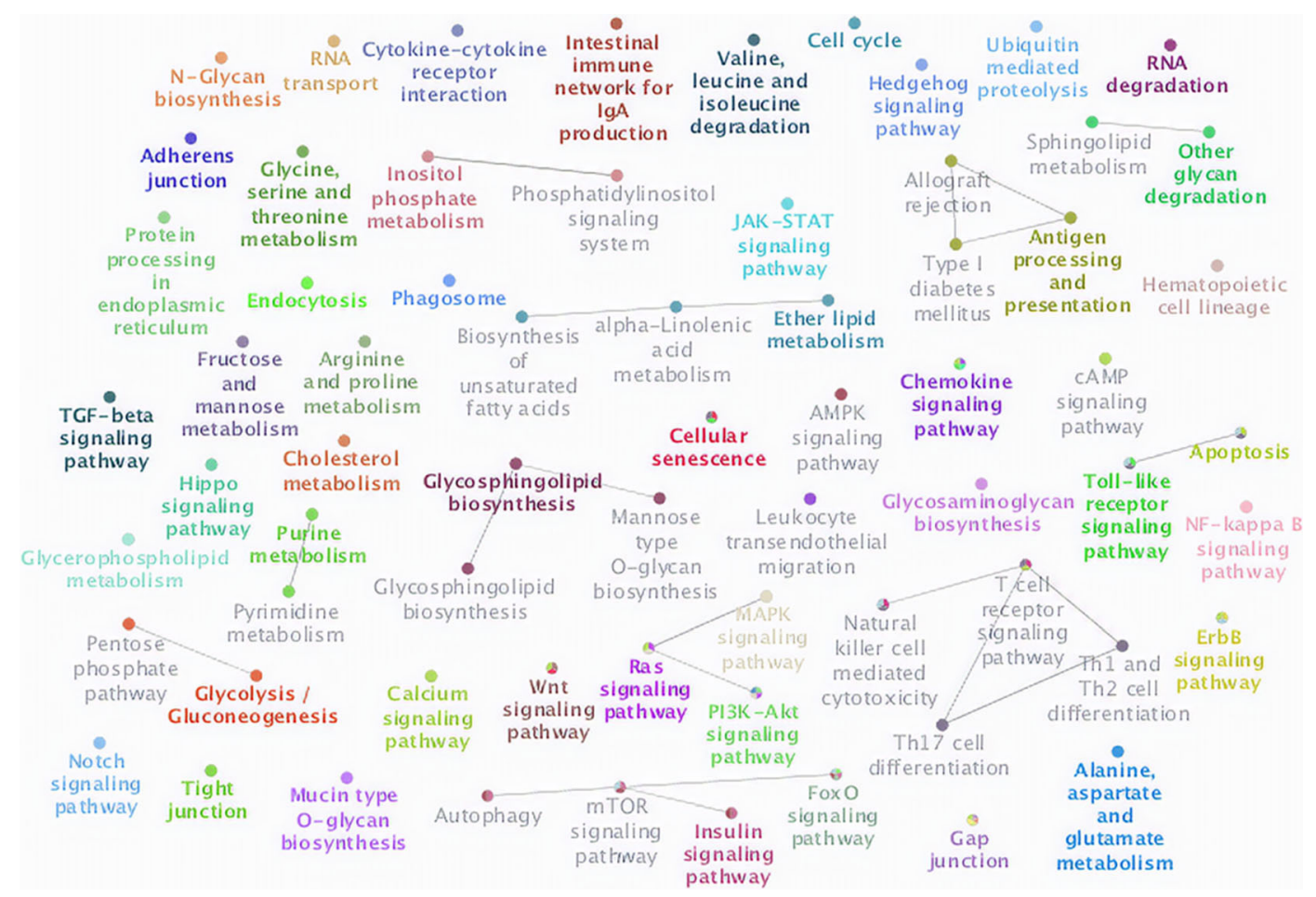

Fig. 3 miR-409-3p target genes pathway analysis. Enrichment of biological functions of predicted and validated miR-409-3p target genes retrieved with miRWalk 3.0 set using Kyoto Encyclopedia of Genes and Genomes (KEGG) analysis in ClueGo. ClueGo revealed correlations among terms as nodes based on their $\mathrm{k}$ score level $(\geq 0.3)$. The most significant term per group is reported and each node represents a biological function. Different colours represent different paths. When the same gene is enriched in different terms, the term is plotted in a variety of colours. The lines represent connections between the nodes and indicate close relatedness of the node's target genes. AMPK, 5'-AMP-activated protein kinase; ERBB, epidermal growth factor receptor; FOXO, forkhead box O; JAK, Janus kinase; MAPK, mitogen-activated protein kinase; mTOR, mammalian target of rapamycin; STAT, signal transducer and activator of transcription; Th1, T helper 1; Th2, T helper 2; Th17, T helper 17

individuals recently diagnosed with type 1 diabetes $(<1$ year of diagnosis) and a healthy non-diabetic (autoantibodynegative) control group. Plasma miR-409-3p levels were reduced in individuals recently diagnosed with type 1 diabetes vs the non-diabetic control group using both the relative and absolute quantification methods (Fig. 7a,b).

A similar miR-409-3p expression pattern was observed in plasma samples obtained from Cohort 2 of individuals with type 1 diabetes with less than 1 year since diagnosis vs a nondiabetic control group (Fig. 7c). Individuals with longstanding type 1 diabetes ( $>2$ years of diagnosis) and individuals with rheumatoid arthritis did not show a reduction in plasma miR409-3p levels vs the non-diabetic control group (Fig. 7c). Instead, an increased level of miR-409-3p was observed in plasma obtained from individuals with rheumatoid arthritis compared with those with type 1 diabetes and the nondiabetic control group (Fig. 7c). No significant correlation was found between plasma miR-409-3p expression and age, BMI, insulin dose, blood glucose level or C-peptide (ESM Fig. 9a-f). However, a negative correlation $(n=-0.52, p=$ 0.04 ) with $\mathrm{HbA}_{1 \mathrm{c}}$ was identified in individuals recently diagnosed with type 1 diabetes (ESM Fig. 9d). Overall, these results suggest that miR-409-3p expression is downregulated 
Fig. 4 Characterisation of miR409-3p expression in NOD lymphocytic infiltrates. (a) Representative images of H\&Estained LCM-captured inflamed islets with an insulitis score of 1,2 or 3; scale bars, $175 \mu \mathrm{m}$. (b) Insulitis scoring analysis. (c-e) Expression analysis of miR-409$3 p$ in plasma samples (c), LCMcaptured immune infiltrates from islets with insulitis score 1-3 (pooled) (d) or LCM-captured islet immune infiltrates separated by insulitis score (score 1 or $2 / 3$ ) (e), from recently diabetic $(n=5)$ and normoglycaemic $(n=5)$ NOD mice. Values are expressed as $2^{-\Delta \mathrm{C}_{\mathrm{t}}}$ plotted on a linear scale (c) or a $\log 10$ scale $(\mathbf{d}, \mathbf{e})$. Data are presented mean \pm SEM. $* p \leq 0.05$, $* * p \leq 0.01$, Mann-Whitney $U$ test. NG, normoglycaemic; a

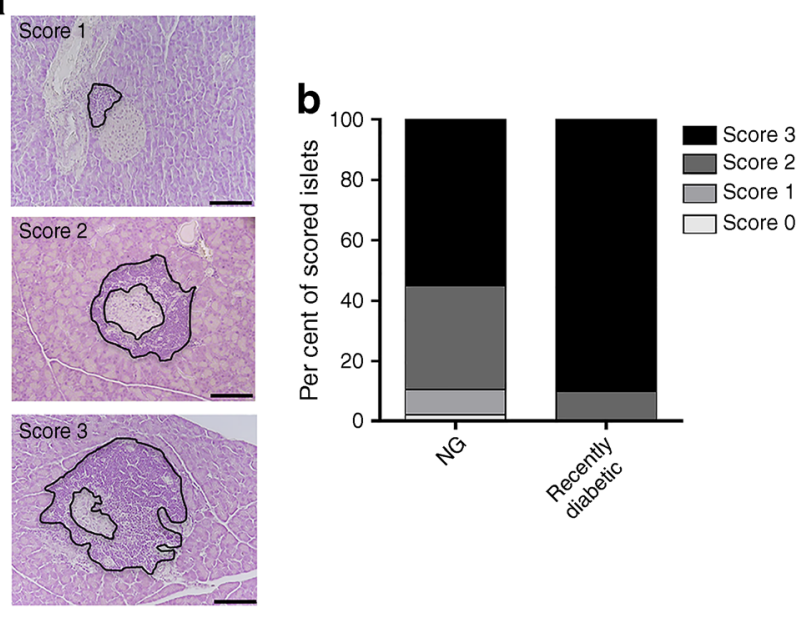

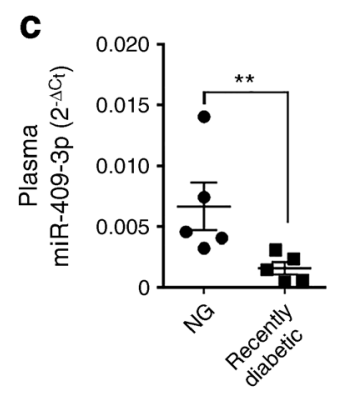
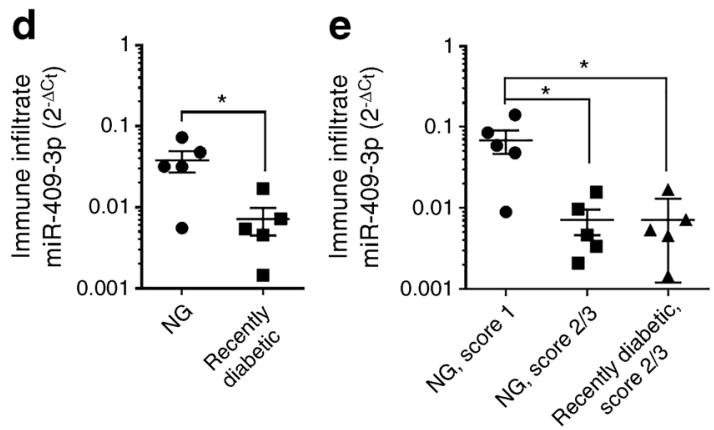

in plasma samples from individuals recently diagnosed with type 1 diabetes vs the non-diabetic control group, and correlates with glycaemic control, similar to that observed in the NOD mouse.

\section{Discussion}

A systematic discovery and characterisation of circulating miRNAs using paired peripheral blood plasma/serum and the diseased tissue are still missing in the field of autoimmune diabetes [30, 31]. However, circulating miRNAs hold great potential as a novel class of non-invasive biomarkers reflecting in situ molecular alterations; the identification of their cellular source could provide new insights into the molecular mechanisms of disease pathogenesis. Thus, circulating miRNAs may be regarded as fingerprints of the affected tissue. We profiled the expression of 384 miRNAs in plasma of recently diabetic and age-matched normoglycaemic NOD mice. We identified five miRNAs (i.e. miR-126a-3p, miR126a-5p, miR-155, miR-204 and miR-409-3p) differentially expressed in plasma of diabetic vs normoglycaemic mice. All were significantly downregulated in plasma of diabetic vs normoglycaemic mice, in line with previous publications in which miRNA expression levels were mainly downregulated [32-34]. Some of these miRNAs identified in plasma of diabetic mice were previously discovered in other autoimmune diseases, such as systemic lupus erythematosus, inflammatory bowel disease and rheumatoid arthritis [35-37]. In the case of human type 1 diabetes, conflicting data have been published regarding the role of these circulating miRNAs. Seyhan et al reported high plasma expression of miR-126 in individuals with type 1 diabetes compared with control participants, while Osipova et al did not find differences in plasma miR-126 levels between paediatric type 1 diabetic participants and age- and sex-matched control participants [15, 38]. Assmann et al documented upregulated expression of miR155 in the plasma of individuals with type 1 diabetes for $<5$ years compared with non-diabetic control participants, while no alterations were detected between the control participants and those with $>5$ years of disease onset [39].

In the present study, miR-409-3p showed consistent reduced expression in paired plasma samples and LCM immune-infiltrated islets of recently diabetic NOD mice, especially in the immune infiltrates belonging to severely infiltrated islets. Moreover, we observed reduced miR-409-3p expression in the plasma of individuals recently diagnosed with type 1 diabetes.

Multiple lines of evidence suggest a significant link between miR-409-3p and immune dysregulation in autoimmune diabetes. First, the miR-409-3p gene is located on human chromosome $14 \mathrm{q} 32$ (or on its mouse homologue 12qF1), a type 1 

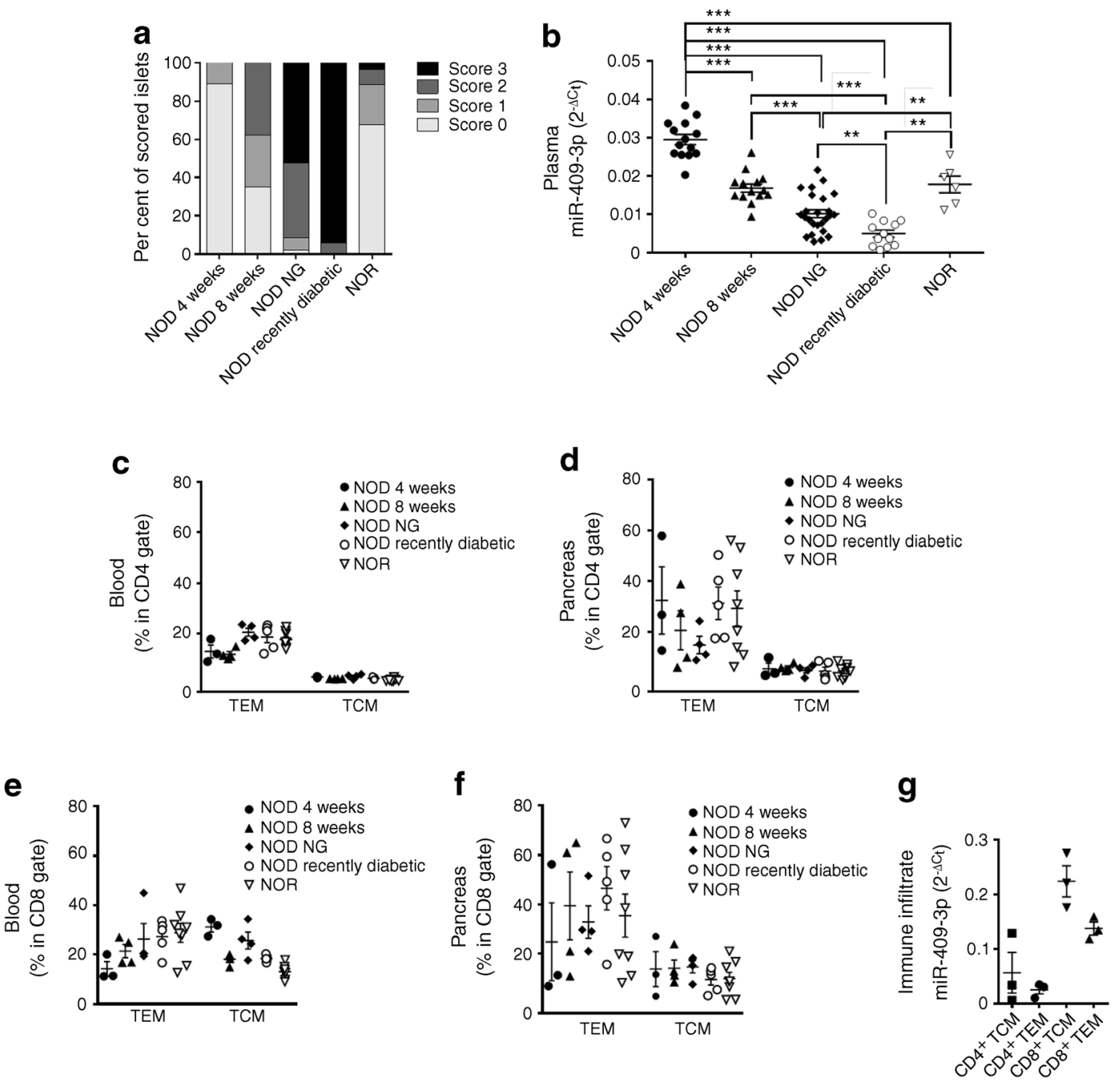

Fig. 5 Circulating miR-409-3p gradually decreases during diabetes development in NOD mice. (a) Pancreatic sections were stained with H\&E and insulitis was scored. (b) Circulating miR-409-3p expression analysis in 4-week-old NOD mice ( $n=14), 8$-week-old NOD mice $(n=14), 17$ - to 25 -week-old normoglycaemic NOD mice $(n=25), 12$ - to 22-week-old recently diabetic NOD mice $(n=12)$ and 21-week-old NOR mice $(n=6)$. Values are reported as $2^{-\Delta C_{t}}$. (c-f) Percentage of CD $44^{+} \mathrm{CD} 62 \mathrm{~L}^{-}$(TEM) and $\mathrm{CD}_{4} 4^{+} \mathrm{CD} 62 \mathrm{~L}^{+}(\mathrm{TCM})$ within $\mathrm{CD}^{+}(\mathbf{c}, \mathbf{d})$ and $\mathrm{CD} 8^{+}(\mathbf{e}, \mathbf{f}) \mathrm{T}$ cell gates in peripheral blood $(\mathbf{c}, \mathbf{e})$ and pancreas $(\mathbf{d}, \mathbf{f})$ of 4-week-old NOD mice $(n=3), 8$-week-old NOD mice $(n=4), 17$ - to 25 -week-old normoglycaemic NOD mice $(n=4), 12$ - to 22 -week-old recently diabetic NOD mice $(n=5)$ and 21-week-old NOR mice $(n=8)$. (g) Expression levels of miR-409-3p in CD4 ${ }^{+}$and CD8+ TEM and TCM cells sorted from the pancreases of 26-week-old normoglycaemic NOD mice. Each dot represents a pool of three mice. Data are reported as $2^{-\Delta C_{t}}$, with values normalised using the small RNA Rnu6 and small nucleolar RNA sno135 and sno202. Data are presented as mean \pm SEM. $* * p \leq 0.01$, *** $p \leq 0.001$, Mann-Whitney $U$ test. NG, normoglycaemic diabetes susceptibility locus [40], which hosts a gene cluster that contains multiple miRNAs that target major type 1 diabetes autoantigens or that are involved in immune-mediated inflammation [41]. Second, our bioinformatics analysis revealed that miR409-3p targets a large number of genes involved in immunerelated pathways (e.g. Pten, Mapk1, Nfatcl, Nfatc2, Ccr5) or in immune metabolism (e.g. Gapdh, Gpil, Pfkl, Pfkfb3, Pfkl). In addition, the phosphoinositide 3-kinase (PI3K)/Akt/forkhead box $\mathrm{O}(\mathrm{FOXO})$ pathway is enriched for miR-409-3p target genes and overlapped with the metabolic pathway genes (e.g. Akt3, Ccnd2, Cdk6, Creb5, Mapk1, Pten, Cptla).

Recent studies have highlighted the importance of miRNAmediated regulation of transcription factors, immune checkpoints and metabolism-triggered molecules during the differentiation of various immune-cell subsets [42-45]. In detail, Socs3 (suppressor of cytokine signalling 3 ) is a direct target of miR-409-3p and is involved in the differentiation of $\mathrm{CD} 8^{+} \mathrm{T}$ cells [46]. Cpt1a, a previously demonstrated regulator of memory $\mathrm{CD}^{+} \mathrm{T}$ cell lipid metabolism and immune response in autoimmune diseases [47], is a conserved predicted target of miR409-3p. Although additional functional studies are needed, these findings already suggest that dysregulated levels of miR-409-3p in plasma and in islet immune infiltrates may be involved in the pathogenesis of type 1 diabetes by inducing aberrant expression of genes involved in metabolic and immune pathways that may modulate the islet microenvironment and regulate the islet- 
a

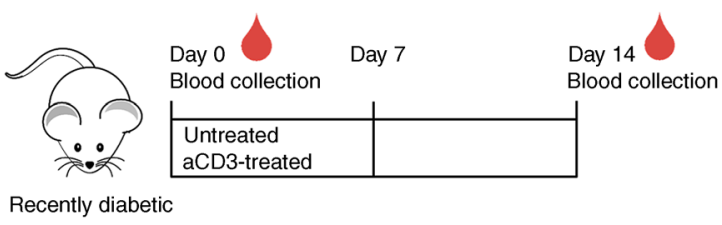

b
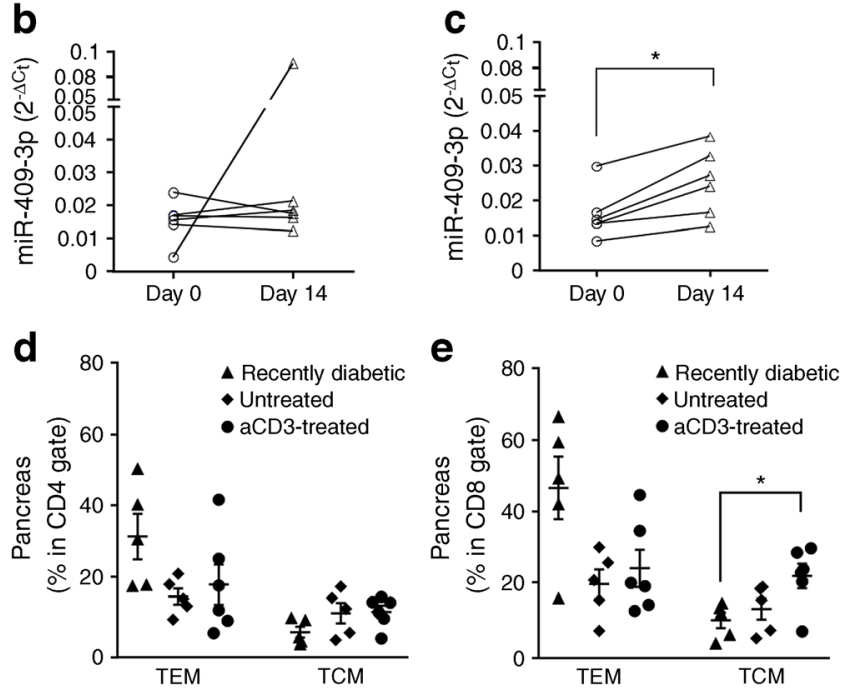

Fig. 6 aCD3 treatment increases plasma circulating levels of miR-4093p. (a) Treatment and sample collection scheme for the administration of cumulative doses (from day 0 to day 7) of aCD 3 in recently diabetic NOD mice followed up to day 14. (b, c) Expression levels of miR-409-3p in plasma collected longitudinally at day 0 (diabetes onset) and at day 14 in untreated $(n=6)(\mathbf{b})$ and aCD3-treated $(n=6)(\mathbf{c})$ groups. Values are expressed as $2^{-\Delta C_{t}}$. The connecting lines indicate paired data from the same NOD mice. (d, e) Percentage of CD44 ${ }^{+} \mathrm{CD} 62 \mathrm{~L}^{-}$(TEM) and $\mathrm{CD} 44^{+} \mathrm{CD}_{2} \mathrm{~L}^{+}(\mathrm{TCM})$ within $\mathrm{CD} 4^{+}(\mathbf{d})$ and $\mathrm{CD} 8^{+}(\mathbf{e}) \mathrm{T}$ cell gates in the pancreas of untreated $(n=5)$ and aCD3-treated $(n=6)$ groups, compared with recently diabetic mice $(n=5)$. Data are presented as mean \pm SEM. $* p \leq 0.05$, Wilcoxon-signed rank test $(\mathbf{b}, \mathbf{c})$ or Mann-Whitney $U$ test $(\mathbf{d}, \mathbf{e})$

derived immune phenotype. A third line of evidence comes from data obtained during the natural course of diabetes progression and after diabetes remission by $\mathrm{aCD} 3$ therapy. While isletspecific inflammation gradually progresses during type 1 diabetes development [48], we observed a gradual reduction in miR409-3p expression in the plasma of prediabetic NOD mice until disease onset. Importantly, plasma levels of miR-409-3p were significantly higher in normoglycaemic diabetes-resistant NOR mice compared with those in age-matched recently diabetic and normoglycaemic NOD mice. It is unlikely that circulating miR409-3p expression levels rely simply on blood glucose levels. Indeed, normoglycaemic NOR mice, having a lower degree of insulitis, had higher plasma miR-409-3p levels compared with age-matched normoglycaemic NOD mice, suggesting that other factors modulate miRNA expression and secretion.

In humans, miR-409-3p has been reported to be expressed in both peripheral $\mathrm{CD}^{+}$and in $\mathrm{CD} 8^{+} \mathrm{T}$ cells [26]. Here, we found that miR-409-3p was also expressed in both islet-associated $\mathrm{CD} 4^{+}$ and $\mathrm{CD}^{+} \mathrm{T}$ cells, with specific enrichment in $\mathrm{CD} 8^{+} \mathrm{T}$ cells
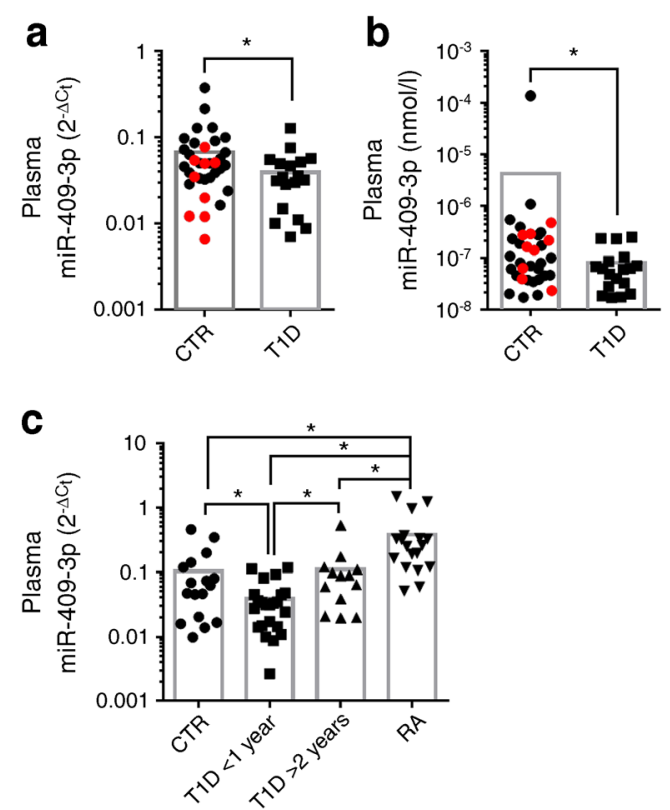

Fig. 7 Relative and absolute miR-409-3p quantification in plasma of non-diabetic control participants and individuals recently diagnosed with type 1 diabetes from different cohorts. (a, b) Relative (reported as $2^{-\Delta \mathrm{C}_{\mathrm{t}}}$ ) (a) and absolute (reported as nmol/1 values interpolated using a synthetic miR-409-3p standard curve) (b) quantification of plasma circulating miR409-3p in non-diabetic individuals (control group; $n=34$ ) and recently diagnosed individuals with type 1 diabetes $(<1$ year since diagnosis; $n=$ 18) (Cohort 1). Red data points indicate autoantibody-negative unaffected family members of individuals with type 1 diabetes. (c) Relative quantification of plasma circulating miR-409-3p in non-diabetic individuals (control; $n=17$ ), recently diagnosed individuals with type 1 diabetes ( $<1$ year of diagnosis; $n=23$ ) and individuals with longstanding type 1 diabetes ( $>2$ years of diagnosis, $n=13$ ) (second, historical cohort [Cohort 2]). Individuals with rheumatoid arthritis $(n=18)$ were also included in the analysis. Data are expressed as $2^{-\Delta \mathrm{C}_{\mathrm{t}}}$ or $\mathrm{nmol} / \mathrm{l}$ and presented as mean values plotted on a $\log _{10}$ scale. $* p \leq 0.05$, Mann-Whitney $U$ test. CTR, control; RA, rheumatoid arthritis; T1D, type 1 diabetes

having a central memory phenotype $\left(\mathrm{CD} 8^{+} \mathrm{TCM}\right.$ cells). Interestingly, we observed a gradual decrease in the frequency of islet-associated $\mathrm{CD} 8^{+} \mathrm{TCM}$ cells during disease progression, coinciding with the reduction in circulating plasma-borne miR409-3p levels. We also found that plasma levels of miR-409-3p were retained following $\mathrm{aCD} 3$ treatment in recently diabetic NOD mice, indicating remodelling of the autoimmune process. Interestingly, we observed that aCD3 therapy increased the frequency of islet-associated $\mathrm{CD} 8^{+} \mathrm{TCM}$ cells, corresponding with the increase in cell-free plasma-borne miR-409-3p levels. Again, miR-409-3p levels were not dependent on blood glucose levels, thus reflecting possible dependence on immune modulation. Concordantly, we and others found that $\mathrm{aCD} 3$ therapy does not block insulitis but rather changes the composition and regulatory balance of the autoimmune infiltrates [17, 18, 28, 49]. Although our data suggest that islet-associated $\mathrm{CD} 8^{+} \mathrm{TCM}$ cells may be the potential secretors of miR-409-3p, we do not claim that the miR409-3p plasma pool derives exclusively from these isletinfiltrating immune cells. Other cell types may also contribute 
to the circulating miR-409-3p pool and specific mechanisms leading to its downregulation in plasma should be further elucidated.

Finally, similarly to what was observed in the NOD mouse model, miR-409-3p levels were lower in plasma of adults recently diagnosed with type 1 diabetes compared with non-diabetic control groups in two independent cohorts. Despite the high overlap of the plasma miR-409-3p levels between individuals recently diagnosed with type 1 diabetes and the healthy non-diabetic participants, the reduction was statistically significant. Future miRNAs analyses could focus on specific plasma components (e.g. exosomes) to reduce this variability. Interestingly, the miR-409-3p reduction was not observed in plasma samples from individuals with longstanding type 1 diabetes, thus suggesting a potential association with disease aggressiveness and insulitis severity. Moreover, miR-409-3p levels correlated with $\mathrm{HbA}_{1 \mathrm{c}}$ at disease onset, further implying a potential association with insulitis severity and progression.

Of relevance, miR-409-3p expression in plasma samples from individuals with rheumatoid arthritis was higher compared with those recently diagnosed with type 1 diabetes and the non-diabetic control group. It should be emphasised that the samples from people with rheumatoid arthritis were not obtained at disease onset and these individuals were taking disease-modifying anti-rheumatic drugs combined with low-dose corticosteroids. If miR409-3p is a circulating biomarker of tissue inflammation, it may not be surprising that individuals with rheumatoid arthritis had higher circulating miR-409-3p levels compared with individuals recently diagnosed with type 1 diabetes. Again, these findings suggest the involvement of miR-409-3p in immune modulation. Still, additional studies will be needed to further elucidate the biological role and cellular origin of miR-409-3p. Although we suggest a potential role for miR-409-3p as a novel biomarker of islet inflammation and immune dysregulation in autoimmune diabetes, we recognise the following limitations: (1) the elevated overlap observed between individuals recently diagnosed with type 1 diabetes and the control group; (2) the absence of a paediatric cohort with type 1 diabetes; and (3) the lack of evidence that specifically demonstrates the cellular origin of miR-409-3p. However, we envision a combined type 1 diabetes biomarker signature including, but not limited to, miR-409-3p alongside other variables comprising additional miRNAs and/or small RNAs.

In conclusion, circulating plasma-borne miR-409-3p may represent a candidate biomarker of islet inflammation in both type 1 diabetic mice and humans and could be potentially useful for serial tracking of islet inflammation or response to immune therapies, such as teplizumab aCD3 therapy, in intervention and prevention trials $[50,51]$.
Acknowledgements We would like to thank L. Dusaer and J. Laureys (Laboratory for Clinical and Experimental Endocrinology, KU Leuven, Belgium) for their technical assistance.

Data availability Data are available from the authors on request.

Funding This project has received funding from the Innovative Medicines Initiative 2 (IMI2) Joint Undertaking under grant agreement no. 115797 (INNODIA). This joint undertaking receives support from the Union's Horizon 2020 research and innovation programme and EFPIA, JDRF and the Leona M. and Harry B. Helmsley Charitable Trust. FD is supported by the Italian Ministry of Research (grant no. $2015373 Z 39$ 007) and by Fondazione Roma. DPC is a PhD fellow of the FWOVlaanderen (Belgium) (11Y6716N).

Duality of interest The authors declare that there is no duality of interest associated with this manuscript.

Contribution statement GV, FM and GS contributed to all aspects of this manuscript, including data acquisition and analysis and drafting and editing the manuscript. DPC, RM and CM were responsible for conception and experimental design, interpretation of the data and editing of the manuscript. FD and CG contributed to conception, experimental design and drafting the manuscript, provided final approval of the submitted manuscript and are guarantors of this work. All authors gave final approval of the version to be published.

\section{References}

1. Finnegan EF, Pasquinelli AE (2013) MicroRNA biogenesis: regulating the regulators. Crit Rev Biochem Mol Biol 48:51-68. https:// doi.org/10.3109/10409238.2012.738643

2. Bartel DP (2009) MicroRNAs: target recognition and regulatory functions. Cell 136:215-233. https://doi.org/10.1016/j.cell.2009. 01.002

3. Wilczynska A, Bushell M (2015) The complexity of miRNAmediated repression. Cell Death Differ 22:22-33. https://doi.org/ 10.1038/cdd.2014.112

4. Guay C, Regazzi R (2017) Exosomes as new players in metabolic organ cross-talk. Diabetes Obes Metab 19(Suppl 1):137-146. https://doi.org/10.1111/dom.13027

5. Kosaka N, Iguchi H, Yoshioka Y et al (2010) Secretory mechanisms and intercellular transfer of microRNAs in living cells. J Biol Chem 285:17442-17452. https://doi.org/10.1074/jbc.M110.107821

6. Fehlmann T, Ludwig N, Backes C et al (2016) Distribution of microRNA biomarker candidates in solid tissues and body fluids. RNA Biol 13:1084-1088. https://doi.org/10.1080/15476286.2016. 1234658

7. Petrovic N, Ergün S, Isenovic ER (2017) Levels of microRNA heterogeneity in cancer biology. Mol Diagn Ther 21:511-523. https://doi.org/10.1007/s40291-017-0285-9

8. Ventriglia G, Nigi L, Sebastiani G, Dotta F (2015) MicroRNAs: novel players in the dialogue between pancreatic islets and immune system in autoimmune diabetes. Biomed Res Int 2015:749734. https://doi.org/10.1155/2015/749734

9. Zeng L, Cui J, Wu H, Lu Q (2014) The emerging role of circulating microRNAs as biomarkers in autoimmune diseases. Autoimmunity 47:419-429. https://doi.org/10.3109/08916934.2014.929667

10. Wang F, Chen C, Wang D (2014) Circulating microRNAs in cardiovascular diseases: from biomarkers to therapeutic targets. Front Med 8:404-418. https://doi.org/10.1007/s11684-014-0379-2 
11. Erener S, Marwaha A, Tan R et al (2017) Profiling of circulating microRNAs in children with recent onset of type 1 diabetes. JCI Insight 2:e89656. https://doi.org/10.1172/jci.insight.89656

12. Samandari N, Mirza AH, Nielsen LB et al (2017) Circulating microRNA levels predict residual beta cell function and glycaemic control in children with type 1 diabetes mellitus. Diabetologia 60: 354-363. https://doi.org/10.1007/s00125-016-4156-4

13. Snowhite IV, Allende G, Sosenko J et al (2017) Association of serum microRNAs with islet autoimmunity, disease progression and metabolic impairment in relatives at risk of type 1 diabetes. Diabetologia 60:1409-1422. https://doi.org/10.1007/s00125-0174294-3

14. Nielsen LB, Wang C, Sørensen K et al (2012) Circulating levels of microRNA from children with newly diagnosed type 1 diabetes and healthy controls: evidence that miR-25 associates to residual betacell function and glycaemic control during disease progression. Exp Diabetes Res 896362:2012. https://doi.org/10.1155/2012/896362

15. Seyhan AA, Nunez Lopez YO, Xie H et al (2016) Pancreasenriched miRNAs are altered in the circulation of subjects with diabetes: a pilot cross-sectional study. Sci Rep 6:31479. https:// doi.org/10.1038/srep31479

16. Guay C, Regazzi R (2013) Circulating microRNAs as novel biomarkers for diabetes mellitus. Nat Rev Endocrinol 9:513-521. https://doi.org/10.1038/nrendo.2013.86

17. Robert S, Gysemans C, Takiishi T et al (2014) Oral delivery of glutamic acid decarboxylase (GAD)-65 and IL10 by Lactococcus lactis reverses diabetes in recent-onset NOD mice. Diabetes 63: 2876-2887. https://doi.org/10.2337/db13-1236

18. Takiishi T, Korf H, Van Belle TL et al (2012) Reversal of autoimmune diabetes by restoration of antigen-specific tolerance using genetically modified Lactococcus lactis in mice. J Clin Invest 122:1717-1725. https://doi.org/10.1172/JCI60530

19. Demeester S, Keymeulen B, Kaufman L et al (2015) Preexisting insulin autoantibodies predict efficacy of otelixizumab in preserving residual $\beta$-cell function in recent-onset type 1 diabetes. Diabetes Care 38:644-651. https://doi.org/10.2337/dc14-1575

20. Bindea G, Galon J, Mlecnik B (2013) CluePedia Cytoscape plugin: pathway insights using integrated experimental and in silico data. Bioinformatics 29:661-663. https://doi.org/10.1093/ bioinformatics/btt019

21. Bindea G, Mlecnik B, Hackl H et al (2009) ClueGO: a Cytoscape plug-in to decipher functionally grouped gene ontology and pathway annotation networks. Bioinformatics 25:1091-1093. https:// doi.org/10.1093/bioinformatics/btp101

22. Magnuson AM, Thurber GM, Kohler RH et al (2015) Population dynamics of islet-infiltrating cells in autoimmune diabetes. Proc Natl Acad Sci USA 112:1511-1516. https://doi.org/10.1073/pnas. 1423769112

23. Willcox A, Richardson SJ, Bone AJ et al (2009) Analysis of islet inflammation in human type 1 diabetes. Clin Exp Immunol 155: 173-181. https://doi.org/10.1111/j.1365-2249.2008.03860.x

24. Coppieters KT, Dotta F, Amirian N et al (2012) Demonstration of islet-autoreactive CD8 T cells in insulitic lesions from recent onset and long-term type 1 diabetes patients. J Exp Med 209:51-60. https://doi.org/10.1084/jem.20111187

25. Arif S, Leete P, Nguyen V et al (2014) Blood and islet phenotypes indicate immunological heterogeneity in type 1 diabetes. Diabetes 63:3835-3845. https://doi.org/10.2337/db14-0365

26. Rossi RL, Rossetti G, Wenandy L et al (2011) Distinct microRNA signatures in human lymphocyte subsets and enforcement of the naive state in CD4+ T cells by the microRNA miR-125b. Nat Immunol 12:796-803. https://doi.org/10.1038/ni.2057

27. Chatenoud L, Primo J, Bach JF (1997) CD3 antibody-induced dominant self tolerance in overtly diabetic NOD mice. J Immunol 158: 2947-2954
28. Wallberg M, Recino A, Phillips J et al (2017) Anti-CD3 treatment up-regulates programmed cell death protein-1 expression on activated effector $\mathrm{T}$ cells and severely impairs their inflammatory capacity. Immunology 151:248-260. https://doi.org/10.1111/imm. 12729

29. Benson RA, Garcon F, Recino A et al (2018) Non-invasive multiphoton imaging of islets transplanted into the pinna of the NOD mouse ear reveals the immediate effect of anti-CD3 treatment in autoimmune diabetes. Front Immunol 9:1006. https://doi.org/10. 3389/fimmu.2018.01006

30. Sebastiani G, Nigi L, Grieco GE et al (2017) Circulating microRNAs and diabetes mellitus: a novel tool for disease prediction, diagnosis, and staging? J Endocrinol Invest 40:591-610. https://doi.org/10.1007/s40618-017-0611-4

31. Zampetaki A, Mayr M (2012) Analytical challenges and technical limitations in assessing circulating miRNAs. Thromb Haemost 108: 592-598. https://doi.org/10.1160/TH12-02-0097

32. Åkerman L, Casas R, Ludvigsson J et al (2018) Serum miRNA levels are related to glucose homeostasis and islet autoantibodies in children with high risk for type 1 diabetes. PLoS One 13: e0191067. https://doi.org/10.1371/journal.pone.0191067

33. Yang M, Ye L, Wang B et al (2015) Decreased miR-146 expression in peripheral blood mononuclear cells is correlated with ongoing islet autoimmunity in type 1 diabetes patients. J Diabetes 7:158165. https://doi.org/10.1111/1753-0407.12163

34. Garcia-Contreras M, Shah SH, Tamayo A et al (2017) Plasmaderived exosome characterization reveals a distinct microRNA signature in long duration type 1 diabetes. Sci Rep 7:5998. https://doi. org/10.1038/s41598-017-05787-y

35. Wang H, Peng W, Ouyang X et al (2012) Circulating microRNAs as candidate biomarkers in patients with systemic lupus erythematosus. Transl Res 160:198-206. https://doi.org/10.1016/j.trsl.2012. 04.002

36. Wang G, Tam L-S, EK-M L et al (2010) Serum and urinary cell-free MiR-146a and MiR-155 in patients with systemic lupus erythematosus. J Rheumatol 37:2516-2522. https://doi.org/10.3899/jrheum. 100308

37. Murata K, Furu M, Yoshitomi $H$ et al (2013) Comprehensive microRNA analysis identifies miR-24 and miR-125a-5p as plasma biomarkers for rheumatoid arthritis. PLoS One 8:e69118. https:// doi.org/10.1371/journal.pone.0069118

38. Osipova J, Fischer D-C, Dangwal S et al (2014) Diabetesassociated microRNAs in pediatric patients with type 1 diabetes mellitus: a cross-sectional cohort study. J Clin Endocrinol Metab 99:E1661-E1665. https://doi.org/10.1210/jc.2013-3868

39. Assmann TS, Recamonde-Mendoza M, Puñales M et al (2018) MicroRNA expression profile in plasma from type 1 diabetic patients: case-control study and bioinformatic analysis. Diabetes Res Clin Pract 141:35-46. https://doi.org/10.1016/j.diabres.2018.03. 044

40. Wallace C, Smyth DJ, Maisuria-Armer M et al (2010) The imprinted DLK1-MEG3 gene region on chromosome 14q32.2 alters susceptibility to type 1 diabetes. Nat Genet $42: 68-71$. https:// doi.org/10.1038/ng.493

41. Abuhatzira L, Xu H, Tahhan G et al (2015) Multiple microRNAs within the $14 \mathrm{q} 32$ cluster target the mRNAs of major type 1 diabetes autoantigens IA-2, IA-2 $\beta$, and GAD65. FASEB J 29:4374- 4383. https://doi.org/10.1096/fj.15-273649

42. Zhang $\mathrm{T}$, Zhang $\mathrm{Z}$, Li F et al (2018) miR-143 regulates memory $\mathrm{T}$ cell differentiation by reprogramming $\mathrm{T}$ cell metabolism. $\mathrm{J}$ Immunol 201:2165-2175. https://doi.org/10.4049/jimmunol. 1800230

43. Essig K, Hu D, Guimaraes JC et al (2017) Roquin suppresses the PI3K-mTOR Signaling pathway to inhibit T helper cell differentiation and conversion of Treg to Tfr cells. Immunity 47 e12:10671082. https://doi.org/10.1016/j.immuni.2017.11.008 
44. Ban YH, Oh S-C, Seo S-H et al (2017) miR-150-mediated Foxo1 regulation programs CD8+ T cell differentiation. Cell Rep 20: 2598-2611. https://doi.org/10.1016/j.celrep.2017.08.065

45. Ouimet M, Ediriweera H, Afonso MS et al (2017) microRNA-33 regulates macrophage autophagy in atherosclerosis. Arterioscler Thromb Vasc Biol 37:1058-1067. https://doi.org/10.1161/ ATVBAHA.116.308916

46. Liu X, Zhou F, Yang Y et al (2018) MiR-409-3p and MiR-1896 cooperatively participate in IL-17-induced inflammatory cytokine production in astrocytes and pathogenesis of EAE mice via targeting SOCS3/STAT3 signaling. Glia 67:101-112. https://doi. org/10.1002/glia.23530

47. Raud B, McGuire PJ, Jones RG et al (2018) Fatty acid metabolism in CD8+ T cell memory: Challenging current concepts. Immunol Rev 283:213-231. https://doi.org/10.1111/imr.12655

48. Christianson SW, Shultz LD, Leiter EH (1993) Adoptive transfer of diabetes into immunodeficient NOD-scid/scid mice. Relative contributions of CD4+ and CD8+ T-cells from diabetic versus prediabetic NOD.NON-Thy-1a donors. Diabetes 42:44-55

49. Baeke F, Van Belle TL, Takiishi T et al (2012) Low doses of antiCD3, ciclosporin A and the vitamin D analogue, TX527, synergise to delay recurrence of autoimmune diabetes in an islet-transplanted NOD mouse model of diabetes. Diabetologia 55:2723-2732. https://doi.org/10.1007/s00125-012-2630-1

50. Herold KC, Bundy BN, Long SA et al (2019) An anti-CD3 antibody, teplizumab, in relatives at risk for type 1 diabetes. $\mathrm{N}$ Engl $\mathrm{J}$ Med 381:603-613. https://doi.org/10.1056/NEJMoa1902226

51. Perdigoto AL, Preston-Hurlburt P, Clark P et al (2019) Treatment of type 1 diabetes with teplizumab: clinical and immunological follow-up after 7 years from diagnosis. Diabetologia 62:655-664. https://doi.org/10.1007/s00125-018-4786-9

Publisher's note Springer Nature remains neutral with regard to jurisdictional claims in published maps and institutional affiliations. 